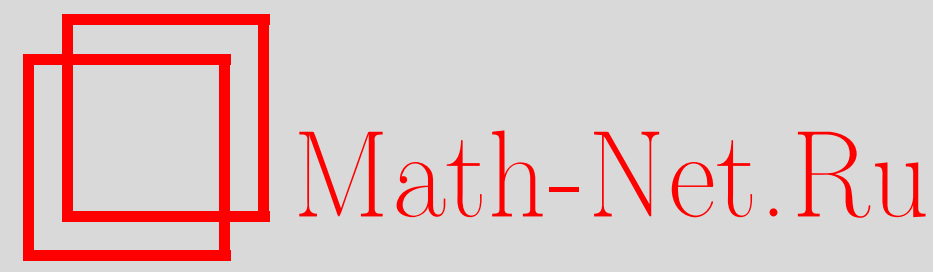

Г. И. Лаптев, Эволюционные уравнения с монотонным оператором и функциональной нелинейностью при производной по времени, Матем. сб., 2000, том 191, номер 9, 43-64

DOI: https://doi.org/10.4213/sm506

Использование Общероссийского математического портала Math-Net.Ru подразумевает, что вы прочитали и согласны с пользовательским соглашением

http://www.mathnet.ru/rus/agreement

Параметры загрузки:

IP : 54.157 .27 .8

26 апреля 2023 г., 14:44:24 


\author{
Г.И. Лаптев
}

\title{
Эволюционные уравнения с монотонным оператором и функциональной нелинейностью при производной по времени
}

\footnotetext{
Изучаются условия разрешимости так назьваемых дважды нелинейных уравнений

$$
A u+\frac{\partial}{\partial t} B u=f, \quad u(0)=u_{0},
$$

где $A$ - монотонный оператор, порождаемьй дифференциальньм вьражением с частными производными высокого порядка, $B$ - оператор, порождаемьй монотонной функцией. Доказана теорема существования решения. Используется метод монотонных операторов, дополненный методом компактности. Даны примеры приложений к параболическим диффференциалњным уравнениям.

Библиограффия: 15 названий.
}

\section{§1. Постановка задачи и формулировка результата}

Работа посвящена исследованию разрешимости уравнений

$$
A u+\frac{\partial}{\partial t} B u=f, \quad u(0)=u_{0},
$$

где $A$ и $B$ - монотонные операторы. Метод монотонных операторов был создан в шестидесятые годы трудами многих математиков и дал возможность изучить широкие классы уравнений с частньми производными высокого порядка эллиптического типа. Определенные итоги метода подведены в монографиях Лионса [1] и Скрыпника [2], а также в обзорной работе Дубинского [3]. Тогда же метод был применен к параболическим уравнениям, которые можно представить в форме $A u+\frac{\partial u}{\partial t}=f$, где $A$ - монотонный оператор. Соответствующие результаты описаны в [1], [4], [5]. При этом в монограффии Лионса [1] неоднократно отмечалось, что на очереди для изучения стоят уравнения вида (1.1), которые называются дважды нелинейными. В качестве приложений можно рассматривать параболические уравнения вида

$$
\sum_{|\alpha| \leqslant m}(-1)^{|\alpha|} D^{\alpha} A_{\alpha}\left(t, x, u, \ldots, D^{m} u\right)+\frac{\partial}{\partial t} B(u)=f .
$$

Здесь применены стандартные обозначения частных производных, но они будут еще разъяснены подробнее в заключительном параграфе работы.

Работа вьполнена при поддержке Российского фонда фундаментальных исследований (грант № 99-01-00045).

$$
\text { (C) Г.И. ЛАптев } 2000
$$


Наиболее изучены к настояшему времени дважды нелинейные уравнения второго порядка по пространственным переменным. Обзор соответствующих работ можно найти в статьях [6]-[9]. По уравнениям высокого порядка результатов несравненно меньше. Выделим работы [10]-[12], в которых строится определенная теория дважды нелинейных уравнений с монотонными операторами, и отметим их отличительные особенности: предполагается, что операторы $A, B$ являются потенциальными и что оператор $B$ компактен относительно оператора $A$. В приложениях к модельным уравнениям вида

$$
\sum_{|\alpha|=m}(-1)^{m} D^{\alpha}\left(\left|D^{\alpha} u\right|^{p-2} D^{\alpha} u\right)+\frac{\partial}{\partial t}\left(|u|^{r-2} u\right)=f
$$

это означает, что степени $p, r$ не могут быть независимыми и связаны соотношением $1 / r>1 / p-m / n$. Ниже предлагается метод, применимый к уравнениям вида (1.3) без ограничений на связь степеней $p, r$ и, разумеется, к более сложным уравнениям. Соответствующие примеры приведены в конще статьи. Предполагается, что оператор $A$ является монотонным достаточно общего вида, а оператор $B$ порождается некоторой функцией $B(u)$. Сформулируем условия на эти операторы более точно.

Пусть $\Omega$ - ограниченная область пространства $\mathbb{R}^{n}, n \geqslant 1$, с достаточно гладкой границей $\partial \Omega$, обеспечивающей применимость теорем вложения Соболева. Далее, используются пространства $L^{p}(\Omega)$ измеримых на $\Omega$ функций, суммируемых со степенью $p, 1<p<\infty$, а также пространства Соболева $W_{p}^{m}(\Omega)$ и $\stackrel{\circ}{W} \underset{p}{m}(\Omega)$, где натуральное $m \geqslant 1$. Пусть $X$ - замкнутое подпространство $W_{p}^{m}(\Omega)$ такое, что $\stackrel{\circ}{W} \underset{p}{m}(\Omega) \subset X \subset W_{p}^{m}(\Omega)$. Напомним, что при указанных условиях $X$ есть сепарабельное рефлексивное банахово пространство. Предполагается, что оно содержит некоторое плотное множество $X_{0}$, удовлетворяюшее включениям $C_{0}^{\infty}(\Omega) \subset X_{0} \subset C(\bar{\Omega})$. Сопряженное к $X$ пространство обозначим $X^{\prime}$. Двойственность задаем символом $(f, u)$, где $f \in X^{\prime}, u \in X$. Если $f, u$ - гладкие функции, например непрерывные, то двойственность определяется формулой

$$
(f, u)=\int_{\Omega} f(x) u(x) d x
$$

Пусть задано число $r \geqslant 2$. Одним из основных считается пространство $Y=$ $L^{r}(\Omega)$. Сопряженным к $Y$ является пространство $Y^{\prime}=L^{r^{\prime}}(\Omega)$, где $r+r^{\prime}=r r^{\prime}$. Так как $r \geqslant 2$, то

$$
Y \subset L^{2}(\Omega) \subset Y^{\prime}
$$

причем вложения плотны и непрерывны. Двойственность между $Y^{\prime}$ и $Y$ обозначается также символом $(f, u)$, где $f \in Y^{\prime}, u \in Y$. Заметим, что для этой двойственности можно использовать формулу (1.4). В частности, если $u \in Y$, то в силу (1.5) определен символ $(u, u)=\int_{\Omega} u^{2}(x) d x$. Пространство $Y$ является сепарабельньп и рефлексивньм. Введем пересечение $Z=X \cap Y$. Оно содержит множество $X_{0}$, которое является плотным как в $X$, так и в $Y$, и становится банаховым пространством, если ввести норму $\|u\|_{Z}=\|u\|_{X}+\|u\|_{Y}$. Это пространство сепарабельно и рефлексивно. Его сопряженное подробно описано в [5; гл. I, теорема 5.13] и имеет вид $Z^{\prime}=X^{\prime}+Y^{\prime}$. 
Далее активно используются пространства абстрактных функций $u(t)$, заданных на отрезке $[0, T]$ и принимаюших значения в некотором банаховом пространстве $S$. Свойства таких пространств изложены, например, в [1] и [5]. Двойственность между элементами $f(t) \in L^{p^{\prime}}\left(0, T ; S^{\prime}\right)$ и $u(t) \in L^{p}(0, T ; S)$ обозначается символом

$$
\langle f, u\rangle=\int_{0}^{T}(f(t), u(t)) d t .
$$

Отметим пространства, играющие далее определяющую роль:

$$
\begin{array}{ll}
\mathscr{X}=L^{p}(0, T ; X) ; & \mathscr{X}^{\prime}=L^{p^{\prime}}\left(0, T ; X^{\prime}\right) ; \\
\mathscr{Y}=L^{r}(0, T ; Y) ; & \mathscr{Y}^{\prime}=L^{r^{\prime}}\left(0, T ; Y^{\prime}\right) ; \\
\mathscr{Z}=\mathscr{X} \cap \mathscr{Y} ; & \mathscr{Z}^{\prime}=\mathscr{X}^{\prime}+\mathscr{Y}^{\prime} .
\end{array}
$$

Записанные здесь равенства для $\mathscr{X}^{\prime}$ и $\mathscr{Y}^{\prime}$ вытекают из [5; гл. IV, теорема 1.14]. Заметим еще, что все эти пространства рефлексивны и сепарабельны.

Сформулируем условия на оператор $A$.

$1 A$. Оператор $A$ определен на пространстве $\mathscr{Z}$, принимает значения в $\mathscr{Z}^{\prime}$ и является непрерывным, ограниченным и монотонным, т.е.

$$
\langle A u-A v, u-v\rangle \geqslant 0, \quad u, v \in \mathscr{Z} .
$$

$2 A$. Оператор $A$ коэрцитивен в следуюшем смысле: если $u \in \mathscr{Z}$, то

$$
\langle A u, u\rangle \geqslant c_{1}\|u\|_{\mathscr{X}}^{p}-C_{1}\|u\|_{\mathscr{Y}}^{r}-C_{2}, \quad c_{1}>0 .
$$

Здесь $p>1, r \geqslant 2$.

Выпишем еще условия на функцию $B(u)$.

$1 B$. Функция $B(u)$ определена на вешественной оси и равномерно монотонна в следуюшем смысле:

$$
(u-v)[B(u)-B(v)] \geqslant c_{2}|u-v|^{r}, \quad c_{2}>0
$$

Степень $r \geqslant 2$ определена в условии $2 A$.

$2 B$. Функция $B(u)$ подчинена следуюшему условию роста:

$$
|B(u)| \leqslant C_{3}\left(1+|u|^{r-1}\right)
$$

3B. Функция $B(u)$ непрерывно дифференцируема. Ее производную обозначим $\beta(u)=B^{\prime}(u)$.

Пусть на $\Omega$ задана измеримая функция $u=u(x)$. Введем оператор $B u=$ $B[u(x)]$. Его областью определения считаем множество $Y=L^{r}(\Omega)$. Далее будут установлены свойства этого оператора. Пока отметим, что он действует непрерывно из $Y$ в $Y^{\prime}$. Именно о нем идет речь в следуюшем утверждении, которое является главным результатом работы.

Теорема 1.1. Пусть выполнены условия $1 A-2 A$ и $1 B-3 B$. Если $u_{0} \in Y u$ $f \in \mathscr{Z}^{\prime}$, то задача (1.1) имеет решение и $(t)$ со следующими свойствами:

(a) $u \in \mathscr{Z} ; A u \in \mathscr{Z}^{\prime}$; 
(b) производная $\frac{\partial}{\partial t} B u(t)$ определена как әлемент из $\mathscr{D}^{\prime}\left(0, T ; L^{r^{\prime}}(\Omega)\right)$ и при этом $\frac{\partial}{\partial t} B u(t) \in \mathscr{Z}^{\prime}$

(c) функиия $u(t)$ удовлетворяет уравнению $A u+\frac{\partial}{\partial t} B u=f$, которое понимается как равенство в пространстве $\mathscr{Z}^{\prime}$;

(d) функиия $U(t)=B u(t) \in C\left([0, T] ; \mathrm{w}-L^{r^{\prime}}(\Omega)\right)$, m.е. она непрерьвна на отрезке $[0, T]$ как абстрактная функиия, принимающая значения в пространстве $L^{r^{\prime}}(\Omega)$, наделенном слабой топологией, и $U(0)=B u_{0}$. В этом смысле понимается начальное условие задачи (1.1).

\section{§ 2. Теорема о компактности}

Здесь собраны вспомогательные утверждения, используемые в основном доказательстве. Через $с$ и $C$ обозначаются, вообще говоря, различные положительные постоянные, которые зависят только от исходных данных рассматриваемой задачи.

Сначала опишем дополнительные свойства функции $B(u)$, введенной ранее. Так как функция $B(u)$ стоит в изучаемом уравнении под знаком производной $\partial / \partial t$, то ее достаточно знать с точностью до постоянной. Поэтому без ограничения общности считаем, что $B(0)=0$. Полагая теперь в условии $1 B$ число $v=0$, приходим к следуюшему утверждению.

ЛЕМма 2.1. Функиия В(и) удовлетворяет соотношению

$$
u B(u) \geqslant c_{2}|u|^{r}, \quad c_{2}>0, \quad r \geqslant 2 .
$$

Если на множестве $\Omega \in \mathbb{R}^{n}$ задана измеримая функция $u=u(x)$, то можно определить оператор $B$ по формуле $B u=B[u(x)]$. Его областью определения будем считать пространство $Y=L^{r}(\Omega)$. Пусть задана абстрактная функция $u(t)$, принимающая значения в $Y$. Применив при каждом $t \in[0, T]$ оператор $B$, получим новую функцию $B u(t)$. Тем самым определен оператор, который будем обозначать той же буквой $B$ и считать определенным на пространстве $\mathscr{Y}=L^{r}(0, T ; Y)$. Для так определенного оператора $B$ справедливы следующие утверждения.

Лемма 2.2. Oператор В действует из пространства $Y$ в $Y^{\prime}$ и является непрерывным, ограниченным и равномерно монотонным:

$$
(B u-B v, u-v) \geqslant c_{2}\|u-v\|_{Y}^{r}, \quad u, v \in Y .
$$

Теми же свойствами он обладает как оператор из У в $\mathscr{Y}^{\prime}$.

ДокАЗАТЕльСтво. Свойство монотонности является непосредственным следствием свойства $1 B$ функции $B(u)$. Аналогично, ограниченность оператора $B$ вытекает из условия $2 B$. Его непрерывность есть следствие ограниченности как оператора суперпозиции, что завершает доказательство леммы.

Введем функцию $\Gamma(u)=\int_{0}^{u} s \beta(s) d s$, где $\beta(u)=B^{\prime}(u)$. Приведем ее свойства.

ЛЕмма 2.3. Функиия Г(и) удовлетворяет следующим условиям роста:

$$
c_{3}|u|^{r} \leqslant \Gamma(u) \leqslant C\left(1+|u|^{r}\right), \quad c_{3}>0 .
$$


ДокАЗАТЕЛЬСтво. Интегрируя по частям, получаем формулу $\Gamma(u)=u B(u)-$ $\int_{0}^{u} B(s) d s$. Применив условие $2 B$ на рост функции $B(u)$, приходим к правому неравенству леммы.

Чтобы вывести левое неравенство, рассмотрим приращение

$$
\Gamma(u)-\Gamma(v)=\int_{v}^{u} s B^{\prime}(s) d s=u B(u)-v B(v)-\int_{v}^{u} B(s) d s .
$$

Последний интеграл оценим сверху, считая для определенности $0<v<u$. Так как функция $B(s)$ монотонно возрастает, то $\int_{v}^{u} B(s) d s \leqslant(u-v) B(u)$. Отсюда для значений $u \leqslant 2 v$ получаем в соответствии с условием $1 B$

$$
\Gamma(u)-\Gamma(v) \geqslant v[B(u)-B(v)] \geqslant(u-v)[B(u)-B(v)] \geqslant c_{2}|u-v|^{r} .
$$

Полагая здесь $v=u / 2$, что допустимо, приходим к оценке $\Gamma(u) \geqslant c_{3}|u|^{r}$. Аналогичная выкладка справедлива для отрицательных значений аргумента, что завершает доказательство леммы.

При использовании метода монотонности важную роль играют выпуклые функции, так как они порождают выпуклые функционалы. Введенные выше функции $B(u)$ и $\Gamma(u)$ выпуклыми быть не обязаны. Однако некоторая их композиция дает выпуклую функцию. Из леммы 2.1 , в частности, следует, что функция $B(u)$ отображает вешественную ось на себя.

По условию $1 B$ эта функция строго монотонна и поэтому имеет обратную функцию $B^{(-1)}$, которая определена на всей числовой оси. Составим композицию $\gamma(U)=\Gamma\left[B^{(-1)}(U)\right]$. Если считать $U=B(u)$, то получаем тождество $\gamma[B(u)]=$ $\Gamma(u)$, которое удобно использовать в таком виде:

$$
\gamma(U)=\Gamma(u), \quad U=B(u)
$$

ЛЕмма 2.4. Функиия $\gamma(U)$ является выпуклой и удовлетворяет условию pocma

$$
|\gamma(U)| \leqslant C\left(1+|U|^{r^{\prime}}\right)
$$

ДокАЗАТЕЛЬСТво. По условию функция $B(u)$ непрерывно дифференцируема и строго монотонно возрастает. Отсюда следует, что ее производная $B^{\prime}(u)$ положительна почти всюду на оси $\mathbb{R}^{1}$. В частности, почти всюду определена функция $[\beta(u)]^{-1}$, и потому верна формула

$$
\gamma^{\prime}(U)=\frac{d \Gamma}{d u}\left(\frac{d B}{d u}\right)^{-1}=u \beta(u)[\beta(u)]^{-1}=u=B^{(-1)}(U) .
$$

Функция $B^{(-1)}(U)$, являясь обратной к непрерывной строго монотонно возрастающей функции, сама непрерывна и монотонно возрастает, что является достаточным для выпуклости функции $\gamma(U)$. Так как функция $\gamma$ определяется через функцию $Г$, то можно использовать лемму 2.3 , что дает

$$
|\gamma(U)|=\left|\Gamma\left[B^{(-1)}(U)\right]\right| \leqslant C\left(1+\left|B^{(-1)}(U)\right|^{r}\right) .
$$


Из леммы 2.1 следует неравенство $|B(u)| \geqslant c_{2}|u|^{r-1}$, которое можно представить в виде $|U| \geqslant c_{2}\left|B^{(-1)}(U)\right|^{r-1}$, откуда $\left|B^{(-1)}(U)\right|^{r} \leqslant C|U|^{r^{\prime}}$. Подставляя это неравенство в (2.2), получаем последнее утверждение леммы.

При изучении уравнений с частными производными, как правило, одного метода монотонности недостаточно, и приходится дополнять его методом компактности. K рассматриваемым здесь дважды нелинейным уравнениям применить известные теоремы о компактности не удается. Это является побудительным мотивом для формулировки и доказательства следующего утверждения.

ТЕорема 2.1 (теорема о компактности). Пусть выполнены сформулированнъе ранее условия на пространства $\mathscr{X}, \mathscr{Y}$, и пусть задана последовательность функиий $u_{N}, N=1,2, \ldots$, со свойствами:

(a) $\sup _{N}\left\|u_{N}\right\| \mathscr{X}=M_{0}<\infty$;

(b) $\sup _{0<\sigma<h} \sup _{N}\left\|u_{N}(t+\sigma)-u_{N}(t)\right\| \mathscr{Y} \rightarrow 0 \quad(h \rightarrow 0)$.

Здесь функиия $u_{N}(t+\sigma)$ считается продоләсенной нулем, если $t+\sigma>T$. Тогда последовательность $u_{N}$ компактна в пространстве $L^{s}(Q)$, где $s=$ $\min (p, r)$ и $Q=\Omega \times(0, T)$. Это значит, что существует подпоследовательность, снова обозначаемая $u_{N}$, и функиия $u \in L^{s}(Q)$ такие, что $u_{N} \rightarrow$ ири $N \rightarrow \infty$ в норме пространства $L^{s}(Q)$. В частности, $u_{N} \rightarrow$ и почти всюду в $Q$.

Доказательство основано на следуюшем утверждении о компактности множества абстрактных функций на отрезке.

ТеОРема 2.2. Пусть Е - сепарабельное банахово пространство, и пусть задана последовательность $u_{N} \in L^{q}(0, T ; E), 1 \leqslant q<\infty, N=1,2, \ldots$, удовлетворяющая условиям:

1) $\sup _{N}\left\|u_{N}\right\|_{L^{q}(0, T ; E)}=M<\infty$;

2) $\sup _{0<\sigma<h} \sup _{N}\left\|u_{N}(t+\sigma)-u_{N}(t)\right\|_{L^{q}(0, T ; E)} \rightarrow 0 \quad(h \rightarrow 0)$;

3) значения интегралов $\int_{\tau}^{t} u_{N}(\sigma) d \sigma$ принадлежсат компактному множеству $K$ пространства $Е$ для всех $0 \leqslant t, \tau \leqslant T$.

Тогда последовательность $u_{N}$ компактна в пространстве $L^{q}(0, T ; E)$.

ДокАЗАТЕльство. Для каждого достаточно малого $h>0$ построим вспомогательную последовательность $u_{N h}(t)=\frac{1}{h} \int_{0}^{h} u_{N}(t+\tau) d \tau$. Убедимся, что новая последовательность компактна в пространстве $C(0, T ; E)$. Оценим прирашения

$$
\left\|u_{N h}(t+\sigma)-u_{N h}(t)\right\|_{E} \leqslant \frac{1}{h} \int_{0}^{h}\left\|u_{N}(t+\tau+\sigma)-u_{N}(t+\tau)\right\|_{E} d \tau .
$$

После замены $t+\tau=\rho$ и применения неравенства Гёльдера с показателями $q$, $q^{\prime}=q /(q-1)$ получаем

$$
\left\|\Delta_{\sigma} u_{N h}\right\|_{E} \leqslant h^{-1 / q}\left(\int_{t}^{t+h}\left\|\Delta_{\sigma} u_{N}(\rho)\right\|_{E}^{q} d \rho\right)^{1 / q} \leqslant h^{-1 / q}\left\|\Delta_{\sigma} u_{N}\right\|_{L^{q}(0, T ; E)} .
$$

Отсюда следует, что

$$
\sup _{N}\left\|\Delta_{\sigma} u_{N h}\right\|_{E} \leqslant h^{-1 / q} \sup _{N}\left\|\Delta_{\sigma} u_{N}\right\|_{L^{q}(0, T ; E)} \rightarrow 0 \quad(\sigma \rightarrow 0) .
$$


Это соотношение показывает, что семейство функций $u_{N h}(t)$ равностепенно непрерывно по $t$ в норме пространства $E$ в соответствии с условием 2) теоремы. Аналогично проверяется, что семейство $u_{N h}(t)$ равномерно по $N$ ограничено в $C(0, T ; E)$. Убедимся еще, что функции $u_{N h}(t)$ принимают значения в компактном множестве пространства $E$. Представим их в виде

$$
u_{N h}(t)=\frac{1}{h} \int_{t}^{t+h} u_{N}(\sigma) d \sigma .
$$

Из условия 3$)$ теоремы следует, что функции $u_{N h}(t)$ принимают значения в множестве $\frac{1}{h} K$, которое является компактным в $E$ для каждого фиксированного $h>0$ вместе с множеством $K$. Подведем итог. При каждом фиксированном $h>0$ последовательность $u_{N h}(t)$ равномерно ограничена в пространстве $C(0, T ; E)$, равностепенно непрерьвна по $t$, определена на компакте $[0, T]$ и принимает значения в компактном множестве пространства $E$. Этого достаточно, чтобы применить обобщенную теорему Арцела, согласно которой множество функций $u_{N h}(t)$ компактно в пространстве $C(0, T ; E)$. Тем более это множество компактно в объемлющем пространстве $L^{q}(0, T ; E)$.

Остается показать, что для всякого $\varepsilon>0$ найдется $h>0$ такое, что множество $\left\{u_{N h}\right\}$ является компактной $\varepsilon$-сетью для исходного множества $\left\{u_{N}\right\}$ в пространстве $L^{q}(0, T ; E)$. Для этого оценим разность

$$
\begin{aligned}
\left\|u_{N h}-u_{N}\right\|_{L^{q}(0, T ; E)}^{q} & =\frac{1}{h^{q}} \int_{0}^{T}\left\|\int_{0}^{h}\left(u_{N}(t+\sigma)-u_{N}(t)\right) d \sigma\right\|_{E}^{q} d t \\
& \leqslant \frac{1}{h^{q}} \int_{0}^{T}\left(\int_{0}^{h}\left\|u_{N}(t+\sigma)-u_{N}(t)\right\|_{E} d \sigma\right)^{q} d t .
\end{aligned}
$$

Применяя к внутреннему интегралу неравенство Гёльдера с показателями $q, q^{\prime}$ и меняя порядок интегралов, получаем

$$
\begin{aligned}
\left\|u_{N h}-u_{N}\right\|_{L^{q}(0, T ; E)}^{q} & \leqslant \frac{1}{h} \int_{0}^{h} \int_{0}^{T}\left\|u_{N}(t+\sigma)-u_{N}(t)\right\|_{E}^{q} d t d \sigma \\
& \leqslant \sup _{0<\sigma<h}\left\|u_{N}(t+\sigma)-u_{N}(t)\right\|_{L^{q}(0, T ; E)}^{q} .
\end{aligned}
$$

Отсюда

$$
\sup _{N}\left\|u_{N h}-u_{N}\right\|_{L^{q}(0, T ; E)} \leqslant \sup _{0<\sigma<h} \sup _{N}\left\|u_{N}(t+\sigma)-u_{N}(t)\right\|_{L^{q}(0, T ; E)}
$$

Согласно условию 2) последнее выражение стремится к нулю вместе с $h \rightarrow 0$. Это значит, что множество $\left\{u_{N}\right\}$ равномерно по $N$ приближается с точностью $\varepsilon$ множеством $\left\{u_{N h}\right\}$, которое компактно в $L^{q}(0, T ; E)$. Следовательно, множество $\left\{u_{N}\right\}$ также компактно в $L^{q}(0, T ; E)$, что доказьвает теорему 2.2 . 
ДоКАЗАТЕЛЬСТво ТЕОРЕмЫ 2.1. Учитывая взаимное расположение чисел $p$ и $r$, рассмотрим два варианта. Пусть сначала $p \leqslant r$. Тогда пространство $L^{r}(Q)$ непрерывно вложено в $L^{p}(Q)$, т.е.

$$
\|u\|_{L^{p}(Q)} \leqslant C_{1}\|u\|_{L^{r}(Q)}, \quad u \in L^{r}(Q),
$$

где постоянная $C_{1}$ не зависит от функции $u \in L^{r}(Q)$. Полагаем в теореме $2.2 q=p$ и $E=L^{p}(\Omega)$. Проверим, что условия этой теоремы тогда выполнены. Заметим, что $L^{q}(0, T ; E)=L^{p}(Q)$, и оценим норму

$$
\left\|u_{N}\right\|_{L^{q}(0, T ; E)}=\left(\int_{0}^{T}\left\|u_{N}(t)\right\|_{L^{p}(\Omega)}^{p} d t\right)^{1 / p} .
$$

По условию пространство $X$ непрерьвно вложено в $L^{p}(\Omega)$ и потому

$$
\left\|u_{N}\right\|_{L^{q}(0, T ; E)} \leqslant C\left(\int_{0}^{T}\left\|u_{N}(t)\right\|_{X}^{p} d t\right)^{1 / p}=C\left\|u_{N}\right\|_{L^{p}(0, T ; X)} \leqslant C M_{0} .
$$

Это доказывает первое условие теоремы 2.2. Чтобы проверить второе, напомним, что $L^{q}(0, T ; E)=L^{p}(Q)$. Применив неравенство $(2.3)$, получим

$$
\left\|u_{N}(t+\sigma)-u_{N}(t)\right\|_{L^{q}(0, T ; E)} \leqslant C_{1}\left\|u_{N}(t+\sigma)-u_{N}(t)\right\| \mathscr{Y},
$$

так как $\mathscr{Y}=L^{r}(Q)$. Остается воспользоваться условием (b) теоремы 2.1, чтобы получить второе условие теоремы 2.2. Проверим третье условие. Для этого оценим

$$
\left\|\int_{\tau}^{t} u_{N}(\sigma) d \sigma\right\|_{X} \leqslant \int_{\tau}^{t}\left\|u_{N}(\sigma)\right\|_{X} d \sigma \leqslant C\left\|u_{N}\right\|_{L^{p}(0, T ; X)} \leqslant C M_{0} .
$$

Это показывает, что значения оцениваемых интегралов лежат в ограниченном множестве $K$ пространства $X$, которое по условию компактно вложено в $L^{p}(\Omega)=E$. Следовательно, значения интегралов $\int_{\tau}^{t} u_{N}(\sigma) d \sigma$ лежат в компактном множестве $K$ пространства $E$, что и требуется. Итак, выполнены все три условия теоремы 2.2, следовательно, последовательность $u_{N}$ компактна в пространстве $L^{p}(0, T ; E)=$ $L^{p}(Q)$, что и утверждалось.

Пусть теперь $p>r$. Тогда справедливо неравенство

$$
\|u\|_{L^{r}(Q)} \leqslant C_{2}\|u\|_{L^{p}(Q)}
$$

где постоянная не зависит от элемента $u \in L^{p}(Q)$. На этот раз полагаем $q=r$ и $E=L^{r}(\Omega)$ в теореме 2.2. Тогда первое условие вытекает из следуюшей цепочки неравенств:

$$
\left\|u_{N}\right\|_{L^{q}(0, T ; E)}=\left\|u_{N}\right\|_{L^{r}(Q)} \leqslant C_{2}\left\|u_{N}\right\|_{L^{p}(Q)} \leqslant C\left\|u_{N}\right\|_{L^{p}(0, T ; X)} \leqslant C M_{0} .
$$

Второе условие теоремы 2.2 в точности совпадает с условием (b) теоремы 2.1. Перейдем к третьему. При рассмотрении случая $p \leqslant r$ было установлено, что интегралы $\int_{\tau}^{t} u_{N}(\sigma) d \sigma$ лежат в компактноммножестве $K$ пространства $L^{p}(\Omega)$. Так как считаем $p>r$, то $L^{p}(\Omega)$ вложено непрерьвно в $L^{r}(\Omega)$, и потому множество $K$ остается компактным в пространстве $L^{r}(\Omega)=E$, что и требуется в третьем условии теоремы 2.2. Тем самым проверены все условия указанной теоремы. Из нее вытекает, что последовательность $u_{N}$ компактна в пространстве $L^{r}(0, T ; E)=L^{r}(Q)$, что завершает доказательство теоремы 2.1 о компактности. 


\section{§3. Конечномерные приближения}

Сначала теорема 1.1 будет доказана при дополнительном условии на функцию $B(u)$.

$4 B$. Производная функции $B(u)$ равномерно положительна:

$$
B^{\prime}(u)=\beta(u) \geqslant c_{0}>0 .
$$

В этом параграфе строятся конечномерные приближения решения задачи (1.1). Напомним, что в пространстве $Z=X \cap Y$ содержится плотное множество $X_{0}$, состоящее из гладких функций, по крайней мере непрерывных в $\bar{\Omega}$. Из множества $X_{0}$ выделим счетную полную в $X$ систему линейно независимых элементов $e_{1}, e_{2}, e_{3}, \ldots$ Линейные комбинации этой системы плотны как в пространстве $X$, так и в $Y=L^{r}(\Omega)$. Ищем приближенные решения задачи (1.1) в виде суммы $u_{N}=\sum_{k=1}^{N} c_{N k}(t) e_{k}$, считая функции $c_{N k}(t)$ непрерывно дифференцируемыми. Для каждого целого $N=1,2,3, \ldots$ составим систему уравнений

$$
\left(\beta\left(u_{N}\right) u_{N}^{\prime}, e_{m}\right)+\left(A u_{N}, e_{m}\right)=\left(f, e_{m}\right), \quad m=1,2, \ldots, N .
$$

Дополним ее начальным условием $u_{N}(0)=u_{N 0}$, предполагая, что последовательность $u_{N 0}=\sum_{k=1}^{N} c_{N k}(0) e_{k}$ сходится к данной функции $u_{0}$ в норме пространства $Y=L^{r}(\Omega)$. Отсюда следует, что последовательность $u_{N}(0)$ равномерно ограничена в $Y$, т.е.

$$
\left\|u_{N}(0)\right\|_{Y} \leqslant C
$$

В развернутом виде система (3.1) имеет вид

$$
\sum_{k=1}^{N}\left(\beta\left(u_{N}\right) e_{k}, e_{m}\right) c_{N k}^{\prime}(t)+\left(A u_{N}, e_{m}\right)=\left(f, e_{m}\right), \quad m=1,2, \ldots, N .
$$

Отсюда непосредственно видно, что получилась система обыкновенных дифференциальных уравнений для функций $c_{N k}(t), k=1,2, \ldots, N$, не разрешенная относительно производных. Учитывая, что $\beta(u)$ - числовая функция, запишем коэффициенты при $c_{N k}^{\prime}(t)$ в виде

$$
\beta_{k m}=\left(\sqrt{\beta\left(u_{N}\right)} e_{k}, \sqrt{\beta\left(u_{N}\right)} e_{m}\right) .
$$

Очевидно, что определитель из элементов $\beta_{k m}$ является определителем Грама системы функций $g_{k}=\sqrt{\beta\left(u_{N}\right)} e_{k}$. Построим линейную комбинацию этих функций

$$
\sum_{k=1}^{N} \alpha_{k} g_{k}=\sqrt{\beta\left(u_{N}\right)} \sum_{k=1}^{N} \alpha_{k} e_{k}
$$

Множитель $\beta\left(u_{N}\right) \geqslant c_{0}>0$ по условию $4 B$, и потому линейная комбинация $\sum_{k=1}^{N} \alpha_{k} g_{k}$ может обратиться в нуль только при условии, что $\sum_{k=1}^{N} \alpha_{k} e_{k}=0$, откуда $\alpha_{k}=0$ для всех $k=1,2, \ldots, N$, так как функции $e_{k}$ по условию линейно независимы. Это показывает, что система функций $g_{k}$ также линейно независима, и потому ее определитель Грама отличен от нуля. А тогда систему дифференциальных уравнений (3.3) можно привести к нормальной форме невырожденньм преобразованием. Напомним еше, что функция $\beta(u)$ и оператор $A$ непрерьвны, что обеспечивает непрерывность коэффициентов системы (3.3) относительно 
неизвестных функций $c_{N k}(t)$. Изложенное выше дает возможность утверждать, что рассматриваемая система дифференциальных уравнений (3.3) разрешима при каждом $N=1,2, \ldots$ по крайней мере на некотором интервале $\left[0, T_{N}\right)$. Мы пришли к следующему утверждению.

Лемма 3.1. Для каждого $N=1,2, \ldots$ система обыкновенных дифференииальных уравнений (3.3) относительно функций $c_{N k}(t)$ разрешима на некотором интервале $\left[0, T_{N}\right)$. При этом функиии $c_{N k}(t)$ как решения системь дифференциальных уравнений непрерывно дифференцируемы. Построенная по ним функиия $u_{N}(t)=\sum_{k=1}^{N} c_{N k}(t) e_{k}$ удовлетворяет системе уравнений (3.1).

Чтобы полученные решения $u_{N}(t)$ продолжить на весь отрезок $[0, T]$, получим для них подходящую оценку. Для этого каждое уравнение системы (3.1) под номером $m=1, \ldots, N$ умножим на функцию $c_{N m}(t)$, сложим все уравнения и результат проинтегрируем по отрезку $[0, t]$, считая $t<T_{N}$. В результате получим тождество

$$
\int_{0}^{t}\left(\beta\left(u_{N}\right) u_{n}^{\prime}, u_{N}\right) d t+\int_{0}^{t}\left(A u_{N}, u_{N}\right) d t=\int_{0}^{t}\left(f, u_{N}\right) d t
$$

Напомним, что функции $e_{k}$, составляющие решение $u_{N}=\sum_{k=1}^{N} c_{N k}(t) e_{k}$, предполагаются непрерывньми на $\bar{\Omega}$. Это дает возможность использовать для вычисления двойственности формулу (1.4). В частности, первое слагаемое тождества (3.4) можно представить в виде

$$
\begin{aligned}
\int_{0}^{t} \int_{\Omega} \beta\left(u_{N}\right) u_{N}^{\prime} u_{N} d x d t & =\int_{\Omega}\left(\int_{u_{N}(0, x)}^{u_{N}(t, x)} u \beta(u) d u\right) d x \\
& =\int_{\Omega} \Gamma\left[u_{N}(t, x)\right] d x-\int_{\Omega} \Gamma\left[u_{N}(0, x)\right] d x
\end{aligned}
$$

Здесь использована функция $\Gamma(v)=\int_{0}^{v} u \beta(u) d u$. Согласно левому неравенству леммы 2.3

$$
\int_{\Omega} \Gamma\left[u_{N}(t, x)\right] d x \geqslant \int_{\Omega} c_{3}\left|u_{N}(t, x)\right|^{r} d x=c_{3}\left\|u_{N}(t)\right\|_{Y}^{r} .
$$

По лемме 2.3 и неравенству (3.2)

$$
\left|\int_{\Omega} \Gamma\left[u_{N}(0, x)\right] d x\right| \leqslant C \int_{\Omega}\left(1+\left|u_{N}(0, x)\right|^{r}\right) d x=C_{1}+C_{2}\left\|u_{N}(0)\right\|_{Y}^{r} \leqslant C
$$

В результате для первого интеграла тождества (3.4) получаем оценку

$$
\int_{0}^{t}\left(\beta\left(u_{N}\right) u_{N}^{\prime}, u_{N}\right) d t \geqslant c_{3}\left\|u_{N}(t)\right\|_{Y}^{r}-C
$$

Введем функцию $v_{N}(t)$, считая ее совпадающей с $u_{N}(t)$ на выбранном отрезке $[0, t]$ и продолженной нулем вне его. Тогда второй интеграл тождества (3.4) можно представить в виде

$$
\int_{0}^{t}\left(A u_{N}, u_{N}\right) d t=\left\langle A v_{N}, v_{N}\right\rangle
$$


что дает возможность использовать условие коэрцитивности $2 A$ оператора $A$ :

$$
\begin{aligned}
\int_{0}^{t}\left(A u_{N}, u_{N}\right) d t & \geqslant c_{1}\left\|v_{N}\right\|_{\mathscr{X}}^{p}-C_{1}\left\|v_{N}\right\|_{\mathscr{Y}}^{r}-C_{2} \\
& =c_{1}\left\|u_{N}\right\|_{L^{p}(0, t ; X)}^{p}-C_{1}\left\|u_{N}\right\|_{L^{r}(0, t ; Y)}^{r}-C_{2} .
\end{aligned}
$$

Оценим еще правую часть тождества (3.4), снова используя введенную выше функцию $v_{N}(t)$ :

$$
\begin{aligned}
\left|\int_{0}^{t}\left(f, u_{N}\right) d t\right| & =\left|\left\langle f, v_{N}\right\rangle\right| \leqslant\|f\|_{\mathscr{Z}^{\prime}} \cdot\left\|v_{N}\right\|_{\mathscr{Z}} \\
& =C\left(\left\|u_{N}\right\|_{L^{p}(0, t ; X)}+\left\|u_{N}\right\|_{L^{r}(0, t ; Y)}\right) \\
& \leqslant \varepsilon\left\|u_{N}\right\|_{L^{p}(0, t ; X)}^{p}+\varepsilon\left\|u_{N}\right\|_{L^{r}(0, t ; Y)}^{r}+C(\varepsilon) .
\end{aligned}
$$

Здесь $\varepsilon$ - любое положительное число. Собирая вместе приведенные выше оценки отдельных слагаемых тождества (3.4), приходим к неравенству

$$
c_{3}\left\|u_{N}(t)\right\|_{Y}^{r}+\left(c_{1}-\varepsilon\right)\left\|u_{N}\right\|_{L^{p}(0, t ; X)}^{p} \leqslant\left(C_{1}+\varepsilon\right) \int_{0}^{t}\left\|u_{N}(s)\right\|_{Y}^{r} d s+C(\varepsilon) .
$$

Введем числовую функцию $y(t)=\left\|u_{N}(t)\right\|_{Y}^{r}$ и выделим из (3.6) неравенство

$$
c_{3} y(t) \leqslant\left(C_{1}+\varepsilon\right) \int_{0}^{t} y(s) d s+C(\varepsilon)
$$

Отсюда по неравенству Гронуолла $[13 ;$ гл. IV,$\S 4$, теорема 2]

$$
\int_{0}^{t} y(t) d t \leqslant C_{1}(\varepsilon)
$$

Подставляя этот результат в неравенство (3.6), получаем

$$
c_{3}\left\|u_{N}(t)\right\|_{Y}^{r}+\left(c_{1}-\varepsilon\right)\left\|u_{N}\right\|_{L^{p}(0, t ; X)}^{p} \leqslant C_{2}(\varepsilon)
$$

Выбирая $\varepsilon>0$ достаточно малым, но фиксированным, приходим к оценкам

$$
\left\|u_{N}\right\|_{L^{\infty}(0, t ; Y)} \leqslant C, \quad\left\|u_{N}\right\|_{L^{p}(0, t ; X)} \leqslant C
$$

где постоянные не зависят от параметра $t \in[0, T]$ и номера $N=1,2, \ldots$ Отсюда следует, что решения $u_{N}(t)$ можно продолжить на весь отрезок $[0, T]$ с сохранением приведенных оценок. Из первой оценки (3.7), в частности, следует, что

$$
\left\|u_{N}\right\|_{\mathscr{Y}}^{r}=\int_{0}^{T}\left\|u_{N}(t)\right\|_{Y}^{r} d t \leqslant T\left\|u_{N}\right\|_{L^{\infty}(0, T ; Y)}^{r} \leqslant C
$$

Объединив ее со второй оценкой (3.7), которую представим в форме $\left\|u_{N}\right\| \mathscr{X} \leqslant C$, получим, что $\left\|u_{N}\right\|_{\mathscr{Z}} \leqslant C$. Напомним еще, что по условию $1 A$ оператор $A$ действует ограниченно из $\mathscr{Z}$ в $\mathscr{Z}^{\prime}$ и потому $\left\|A u_{N}\right\|_{\mathscr{Z}^{\prime}} \leqslant C$. Все указанные оценки объединим в следующем утверждении. 
Лемма 3.2. Решения системь (3.1) определень на всем отрезке $[0, T] и$ удовлетворяют оценкам

$$
\begin{gathered}
\max _{t}\left\|u_{N}(t)\right\|_{Y} \leqslant C, \quad\left\|u_{N}\right\| \mathscr{Y} \leqslant C \\
\left\|u_{N}\right\|_{\mathscr{Z}} \leqslant C, \quad\left\|A u_{N}\right\|_{\mathscr{Z}^{\prime}} \leqslant C .
\end{gathered}
$$

Здесь постояннье не зависят от номера $N=1,2, \ldots$.

В дальнейшем существенную роль играет последовательность $U_{N}=B u_{N}$. Отметим некоторые ее свойства.

ЛЕмма 3.3. Последовательность $U_{N}$ равномерно ограничена как в пространстве $L^{\infty}\left(0, T ; Y^{\prime}\right)$, так и в пространстве $\mathscr{Y}^{\prime}$. Справедлива формула

$$
\left\langle U_{N}^{\prime}, u_{N}\right\rangle=\int_{\Omega} \Gamma\left[u_{N}(T, x)\right] d x-\int_{\Omega} \Gamma\left[u_{N}(0, x)\right] d x .
$$

ДоказАтЕльство. Напомним, что по лемме 2.2 оператор $B: Y \rightarrow Y^{\prime}$ ограничен, и потому из первой оценки леммы 3.2 вытекает, что $\max _{t}\left\|B u_{N}\right\|_{Y^{\prime}} \leqslant C$. Это соответствует равномерной ограниченности последовательности $U_{N}$ в пространстве $L^{\infty}\left(0, T ; Y^{\prime}\right)$. Аналогично, из второй оценки леммы 3.2 вытекает неравенство $\left\|B u_{N}\right\|_{\mathscr{Y}^{\prime}} \leqslant C$, что означает ограниченность последовательности $U_{N}$ в пространстве $\mathscr{Y}^{\prime}$. Остается убедиться в заключительном тождестве леммы. Так как $U_{N}^{\prime}=\beta\left(u_{N}\right) u_{N}^{\prime}$, то оно фактически совпадает с тождеством (3.5), в котором следует считать $t=T$. Это завершает доказательство леммы.

Перейдем к более тонким свойствам последовательности $U_{N}$. Пусть $t, t+h \in$ $[0, T]$. Рассмотрим прирашения $\Delta_{h} U_{N}=U_{N}(t+h)-U_{N}(t)$. Введем конечномерные пространства $E_{N}$, натянутые на первые $N$ элементов базиса $e_{1}, \ldots, e_{N}$. Справедливо следуюшее утверждение.

Лемма 3.4. Пусть $e \in E_{N}$. Тогдa

$$
\left|\left(\Delta_{h} U_{N}, e\right)\right| \leqslant C h^{\alpha}\|e\|_{Z}, \quad \alpha=\min \left(\frac{1}{p}, \frac{1}{r}\right) .
$$

Здесь постоянная не зависит от $h$ и номера $N=1,2, \ldots$.

ДокАЗАТЕЛЬСтво. Используем очевидное представление

$$
\left(\Delta_{h} U_{N}, e\right)=\int_{t}^{t+h}\left(U_{N}^{\prime}(\tau), e\right) d \tau
$$

Согласно введенным ранее обозначениям

$$
U_{N}^{\prime}=\frac{\partial}{\partial t} B\left(u_{N}\right)=B^{\prime}\left(u_{N}\right) u_{N}^{\prime}=\beta\left(u_{N}\right) u_{N}^{\prime} .
$$

В результате тождество (3.1) для всякого элемента $e \in E_{N}$ можно представить в виде

$$
\left(U_{N}^{\prime}, e\right)+\left(A u_{N}, e\right)=(f, e), \quad e \in E_{N} .
$$


Выразим отсюда первое слагаемое и подставим в формулу для $\left(\Delta_{h} U_{N}, e\right)$, что дает

$$
\left(\Delta_{h} U_{N}, e\right)=\int_{t}^{t+h}(f, e) d \tau-\int_{t}^{t+h}\left(A u_{N}, e\right) d \tau .
$$

Введем функцию $v(\tau)$, считая ее равной элементу $е$ для каждого $\tau \in[t, t+h]$ и продолженной нулем вне этого отрезка. Тогда равенство (3.9) можно представить в виде

$$
\left(\Delta_{h} U_{N}, e\right)=\langle f, v\rangle-\left\langle A u_{N}, v\right\rangle .
$$

Оценим каждое слагаемое правой части:

$$
\begin{gathered}
|\langle f, v\rangle| \leqslant\|f\|_{\mathscr{Z}^{\prime}} \cdot\|v\|_{\mathscr{Z}} \leqslant C\|v\|_{\mathscr{Z}}, \\
\left|\left\langle A u_{N}, v\right\rangle\right| \leqslant\left\|A u_{N}\right\|_{\mathscr{Z}^{\prime}} \cdot\|v\|_{\mathscr{Z}} \leqslant C\|v\|_{\mathscr{Z}} .
\end{gathered}
$$

Здесь использована лемма 3.2. В результате из (3.10) следует неравенство

$$
\left|\left(\Delta_{h} U_{N}, e\right)\right| \leqslant C\|v\|_{\mathscr{Z}} .
$$

Последняя норма легко вычисляется с учетом определения функции $v(t)$ :

$$
\|v\|_{\mathscr{Z}}=\|v\|_{\mathscr{X}}+\|v\|_{\mathscr{Y}}=h^{1 / p}\|e\|_{X}+h^{1 / r}\|e\|_{Y} \leqslant h^{\alpha}\|e\|_{Z}, \quad \alpha=\min \left(\frac{1}{p}, \frac{1}{r}\right) .
$$

Подставляя это неравенство в (3.11), получаем утверждение леммы.

ЛЕмма 3.5. Пусть $h$ - фиксированное число, причем $|h| \leqslant 1$. Введем функцию $u_{N}(t+h)$, считая ее продолженной нулем вне отрезка $[0, T], u$ определим приращения $\Delta_{h} u_{N}=u_{N}(t+h)-u_{N}(t)$. Тогда

$$
\left\|\Delta_{h} u_{N}\right\|_{\mathscr{Y}} \leqslant C h^{\beta}, \quad \beta=\frac{\alpha}{r} .
$$

Здесъ постоянная не зависит от $h$ и номера $N=1,2, \ldots$.

ДокАЗАТЕЛЬСтво. Рассмотрим произведение

$$
\left(\Delta_{h} U_{N}, \Delta_{h} u_{N}\right)=\left(U_{N}(t+h)-U_{N}(t), u_{N}(t+h)-u_{N}(t)\right) .
$$

Напомним, что $U_{N}=B u_{N}$, поэтому

$$
\left(\Delta_{h} U_{N}, \Delta_{h} u_{N}\right)=\left(B u_{N}(t+h)-B u_{N}(t), u_{N}(t+h)-u_{N}(t)\right) .
$$

Используем лемму 2.2 о равномерной монотонности оператора $B: Y \rightarrow Y^{\prime}$, что дает оценку снизу

$$
\left(\Delta_{h} U_{N}, \Delta_{h} u_{N}\right) \geqslant c_{2}\left\|u_{N}(t+h)-u_{N}(t)\right\|_{Y}^{r} .
$$

Элемент $u_{N}(t)=\sum_{k=1}^{N} c_{N k}(t) e_{k}$ при каждом $t \in[0, T]$ принадлежит пространству $E_{N}$, и потому к рассматриваемому произведению можно применить лемму 3.4 , полагая $e=\Delta_{h} u_{N}(t)$. Это дает оценку сверху

$$
\left|\left(\Delta_{h} U_{N}, \Delta_{h} u_{N}\right)\right| \leqslant C h^{\alpha}\left\|\Delta_{h} u_{N}(t)\right\|_{Z} \leqslant 2 C h^{\alpha}\left\|u_{N}(t)\right\|_{Z} .
$$

Объединяя оценки снизу и сверху, получаем

$$
\mathrm{c}_{2}\left\|u_{N}(t+h)-u_{N}(t)\right\|_{Y}^{r} \leqslant C h^{\alpha}\left\|u_{N}(t)\right\|_{Z} .
$$

Это неравенство интегрируем по отрезку $[0, T]$ и используем лемму 3.2 , что дает

$$
c_{2} \int_{0}^{T}\left\|u_{N}(t+h)-u_{N}(t)\right\|_{Y}^{r} d t \leqslant C h^{\alpha} .
$$

Это есть утверждение леммы. 
Лемма 3.6. Найдется функиия $u(t, x) \in \mathscr{Z} \cap L^{\infty}(0, T ; Y)$ со следующими свойствами: из последовательности и м можно извлечь подпоследовательность, снова обозначаемую через $u_{N}$, такую, что при $N \rightarrow \infty$

(a) $u_{N} \rightarrow$ и почти всюду в $Q$;

(b) $U_{N} \rightarrow U=$ Ви почти всюду в $Q$.

ДокАЗАТЕЛЬство. Пункт (а) есть следствие леммы 3.5 и теоремы 2.1 о компактности. Пункт (b) является следствием предыдущего, так как $U_{N}=B u_{N}$, где $B$ - непрерывная функция. Принадлежность $u \in \mathscr{Z} \cap L^{\infty}(0, T ; Y)$ вытекает из оценок леммы 3.2 .

ЛЕмма 3.7. Из последовательности $u_{N}$ можно извлечь подпоследовательность, снова обозначаемую через $u_{N}$, такую, что при $N \rightarrow \infty$ :

(a) $u_{N} \rightarrow$ слабо в $\mathscr{Z}$;

(b) $A u_{N} \rightarrow W$ слабо в $\mathscr{Z}^{\prime}$;

(c) $U_{N} \rightarrow U=B u *$-слабо в $L^{\infty}\left(0, T ; Y^{\prime}\right)$, m.e. $\left\langle U_{N}, v\right\rangle \rightarrow\langle U, v\rangle$ для любого элемента $v \in L^{1}(0, T ; Y) ;$ при этом $U \in L^{\infty}\left(0, T ; Y^{\prime}\right)$.

ДокаЗАТЕльство. По лемме $3.2\left\|u_{N}\right\|_{\mathscr{Z}} \leqslant C$. Так как пространство $\mathscr{Z}$ сепарабельно и рефлексивно, то найдется элемент $u^{(1)} \in \mathscr{Z}$ такой, что $u_{N} \rightarrow u^{(1)}$ слабо в $\mathscr{Z}$. Одновременно по лемме $3.6 u_{N} \rightarrow u$ почти всюду в $Q$, причем $u \in \mathscr{Z}$. Совпадение двух указанных пределов установлено, например, в [5; гл. II, лемма 1.19]. Тем самым $u^{(1)}=u$, что доказывает п. (а). Аналогично п. (с) следует из лемм 3.3 и 3.6 . Поскольку пространство $\mathscr{Z}^{\prime}$ также сепарабельно и рефлексивно, то найдется элемент $W \in \mathscr{Z}^{\prime}$ такой, что $A u_{N} \rightarrow W$ слабо в $\mathscr{Z}^{\prime}$ при $N \rightarrow \infty$. Это доказывает п. (b) и завершает доказательство леммы.

Обозначим через $\mathscr{Y}_{N}$ пространство функций вида $\sum_{k=1}^{N} d_{k}(t) e_{k}, d_{k} \in C^{1}[0, T]$. И пусть $\mathscr{Y}_{0}=\bigcup_{k=1}^{\infty} \mathscr{Y}_{N}$. Очевидно, что $\mathscr{Y}_{0}$ содержится в пространстве $\mathscr{Z}$ и плотно в нем.

ЛЕмма 3.8. Для функиии $U(t)=B u(t)$, построенной в лемме 3.6, определена производная $U^{\prime}(t)=\frac{\partial}{\partial t} B u(t)$ как әлемент пространства $\mathscr{D}^{\prime}\left(0, T ; Y^{\prime}\right)$.

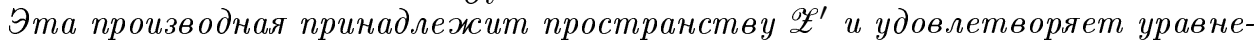
нию

$$
U^{\prime}+W=f
$$

которое понимается как равенство в пространстве $\mathscr{Z}^{\prime}$ и где әлемент $W$ взят из леммы 3.7. Для каэсдой функиии $v \in \mathscr{Y}_{0}$ при $N \rightarrow \infty$ справедливо предельное соотношение

$$
\left\langle U_{N}^{\prime}, v\right\rangle \rightarrow\left\langle U^{\prime}, v\right\rangle, \quad v \in \mathscr{Y}_{0}
$$

ДокаЗАТЕльство. Пусть $\varphi \in \mathscr{D}(0, T) ; e \in Y$. Полагаем $v=\varphi^{\prime}(t) e$. Очевидно, что $v \in L^{1}(0, T ; Y)$ и потому согласно лемме $3.7($ с $)$ при $N \rightarrow \infty$

$$
\left\langle U_{N}, \varphi^{\prime}(t) e\right\rangle \rightarrow\left\langle U, \varphi^{\prime}(t) e\right\rangle .
$$

Это соотношение означает, что определены производные $U_{N}^{\prime}$ и $U^{\prime}$ как элементы пространства $\mathscr{D}^{\prime}\left(0, T ; Y^{\prime}\right)$, причем для каждой функции $v=\varphi(t) e$, где $\varphi \in \mathscr{D}(0, T)$ :

$$
\left\langle U_{N}^{\prime}, v\right\rangle \rightarrow\left\langle U^{\prime}, v\right\rangle, \quad N \rightarrow \infty
$$


Ясно, что это предельное соотношение распространяется на функции вида $v=$ $\sum_{k=1}^{M} d_{k}(t) e_{k}$, если $d_{k} \in \mathscr{D}(0, T)$.

Пусть $v \in \mathscr{Y}_{0}$, т.е. $v=\sum_{k=1}^{M} d_{k}(t) e_{k}$. Тогда $v(t) \in E_{N}$ для каждого $t \in[0, T]$ и для номеров $N \geqslant M$. Это значит, что для указанных номеров $N$ можно использовать тождество (3.8), полагая $e=v(t), t \in[0, T]$, что после интегрирования по отрезку $[0, T]$ дает

$$
\left\langle U_{N}^{\prime}, v\right\rangle+\left\langle A u_{N}, v\right\rangle=\langle f, v\rangle, \quad v \in \mathscr{Y}_{0}
$$

В элементе $v \in \mathscr{Y}_{0}$ выберем функции $d_{k} \in \mathscr{D}(0, T)$. Тогда можно использовать предельное соотношение (3.12), которое с учетом соотношения $\left\langle A u_{N}, v\right\rangle \rightarrow\langle W, v\rangle$, установленного в лемме 3.7 , приводит к равенству

$$
\left\langle U^{\prime}, v\right\rangle+\langle W, v\rangle=\langle f, v\rangle
$$

Учитывая плотность множества функций $v \in \mathscr{Y}_{0}$ указанной структуры в пространстве $\mathscr{Z}$, получаем соотношение $U^{\prime}+W=f$, которое доказывает п. (а). Отсюда следует, в частности, что $U^{\prime} \in \mathscr{Z}^{\prime}$. Остается доказать соотношение (b). Оно уже известно для специального класса функций $v=\sum_{k=1}^{M} d_{k}(t) e_{k}$, если $d_{k} \in \mathscr{D}(0, T)$, и потому задача состоит в расширении класса допустимых множителей $v$. Пусть $v \in \mathscr{Y}_{0}$, тогда его можно использовать в тождестве (3.13). Одновременно $v$ можно подставить в равенство (3.14). Вычитая одно из другого, получаем

$$
\left\langle U_{N}^{\prime}, v\right\rangle-\left\langle U^{\prime}, v\right\rangle=\langle W, v\rangle-\left\langle A u_{N}, v\right\rangle, \quad v \in \mathscr{Y}_{0}
$$

Так как правая часть стремится к нулю при $N \rightarrow \infty$ в силу леммы $3.7(\mathrm{~b})$, то это же верно для левой части, что доказывает п. (b) и завершает доказательство леммы.

ЛЕмма 3.9. Из последовательности $U_{N}$ можсно выбрать подпоследовательность, снова обозначаемую через $U_{N}$, для которой справедливы следующие соотношения:

(a) $U_{N}(0) \rightarrow U(0)$ в $Y^{\prime}$;

(b) $U(0)=B u_{0}$;

(c) $U_{N}(T) \rightarrow U(T)$ слабо в $Y^{\prime}$.

ДоказАТЕЛьство. Согласно определению $U_{N}=B u_{N}$. По построению $u_{N}(0) \rightarrow u_{0}$ в $Y$. Оператор $B: Y \rightarrow Y^{\prime}$ непрерьвен по лемме 2.2 и потому $B u_{N}(0) \rightarrow B u_{0}$ в пространстве $Y^{\prime}$, т.е. $U_{N}(0) \rightarrow B u_{0}$ в $Y^{\prime}$. Остается убедиться, что $B u_{0}=U(0)$. По лемме $3.3\left\|U_{N}(0)\right\|_{Y^{\prime}} \leqslant C$ и потому можно считать, что $U_{N}(0) \rightarrow W_{0}$ слабо в $Y^{\prime}$. Пусть $E_{0}=\bigcup_{N=1}^{\infty} E_{N}$. Введем функцию $v=(T-t) e$, полагая $e \in E_{0}$. Прямым выгислением убеждаемся, что для достаточно больших номеров $N$

$$
\left\langle U_{N}^{\prime}, v\right\rangle=\int_{0}^{T}\left(U_{N}(t), e\right) d t-T\left(U_{N}(0), e\right) .
$$

Очевидно, что $v \in \mathscr{Y}_{0}$, поэтому можно использовать лемму $3.8(\mathrm{~b})$, с помощью которой в пределе при $N \rightarrow \infty$ получаем тождество

$$
\left\langle U^{\prime}, v\right\rangle=\langle U, e\rangle-T\left(W_{0}, e\right)
$$


Вычитая его из аналогичного тождества

$$
\left\langle U^{\prime},(T-t) e\right\rangle=\langle U, e\rangle-T(U(0), e)
$$

получаем равенство $\left(W_{0}-U(0), e\right)=0, e \in E_{0}$. Так как множество $E_{0}$ плотно в $Y$, то $W_{0}=U(0)$. Вспоминая определение элемента $W_{0}$, приходим к соотношению: $U_{N}(0) \rightarrow U(0)$ слабо в $Y^{\prime}$. Одновременно $U_{N}(0) \rightarrow B u_{0}$ в $Y^{\prime}$, как было установлено ранее. Следовательно, $U(0)=B u_{0}$ и одновременно $U_{N}(0) \rightarrow U(0)$ в $Y^{\prime}$. Это доказывает пп. (a) и (b). Если провести аналогичные выгисления для функции $v=t e, e \in E_{0}$, то придем к соотношению: $U_{N}(T) \rightarrow U(T)$ слабо в $Y^{\prime}$. Это завершает доказательство леммы.

ЛЕмма 3.10. Функиия $U(t)=B u(t)$ непрерывна на отрезке $[0, T]$ как абстрактная функция, принимающая значения в пространстве $Y^{\prime}$, наделенном слабой топологией, и при этом $U(0)=B u_{0}$.

ДокАЗАтЕльство. Пусть $t, t+h \in[0, T]$. Введем скалярную функцию $(U(t), e)$, считая $e \in Z$. Ее приращение представим в виде

$$
\left(\Delta_{h} U, e\right)=(U(t+h)-U(t), e)=\int_{t}^{t+h}\left(U^{\prime}(\tau), e\right) d \tau
$$

Учитывая равенство $U^{\prime}=f-W$ леммы 3.8 и используя оценки леммы 3.4 , приходим к неравенству

$$
\left|\left(\Delta_{h} U, e\right)\right| \leqslant C h^{\alpha}\|e\|_{Z}, \quad \alpha=\min \left(\frac{1}{p}, \frac{1}{r}\right) .
$$

Отсюда следует непрерывность введенной функции, что соответствует непрерывности функции $U(t)$ в слабой топологии пространства $Y^{\prime}$. Равенство $U(0)=B u_{0}$ было установлено в лемме $3.9(\mathrm{~b})$.

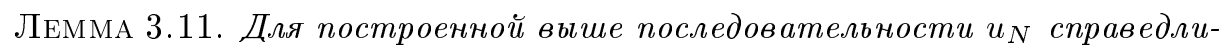
вы следующие предельные соотношения:

$$
\begin{aligned}
& \lim _{N \rightarrow \infty} \int_{\Omega} \Gamma\left[u_{N}(0, x)\right] d x=\int_{\Omega} \Gamma[u(0, x)] d x \\
& \underset{N \rightarrow \infty}{\lim } \int_{\Omega} \Gamma\left[u_{N}(T, x)\right] d x \geqslant \int_{\Omega} \Gamma[u(T, x)] d x .
\end{aligned}
$$

ДокАЗАТЕЛЬСТво. Используем функцию $\gamma(U), U=B(u)$, определенную в $\S 2$, и тождества (2.1):

$$
\gamma(U)=\Gamma(u), \quad U=B(u)
$$

В новых обозначениях соотношение (a) примет вид

$$
\lim _{N \rightarrow \infty} \int_{\Omega} \gamma\left[U_{N}(0, x)\right] d x=\int_{\Omega} \gamma[U(0, x)] d x .
$$

Введем функционал

$$
F(U)=\int_{\Omega} \gamma[U(x)] d x
$$


считая его определенньм на пространстве $Y^{\prime}$. Лемма 2.4 показывает, что функционал $F$ непрерывен и локально ограничен на $Y^{\prime}$ [14; гл. II, $\S 5$, п. 5.1, пример 5.1]. По лемме $3.9 U_{N}(0) \rightarrow U(0)$ в $Y^{\prime}$, следовательно, $F\left[U_{N}(0)\right] \rightarrow F[U(0)]$ при $N \rightarrow \infty$. Это в точности соответствует равенству (3.15), что доказывает п. (a) леммы.

Перейдем к (b). Снова используя функцию $\gamma(U)$, запишем (b) в виде

$$
\varliminf_{N \rightarrow \infty} \int_{\Omega} \gamma\left[U_{N}(T, x)\right] d x \geqslant \int_{\Omega} \gamma[U(T, x)] d x .
$$

В терминах функционала $F(U)$ соотношение (3.16) имеет вид:

$$
\varliminf_{N \rightarrow \infty} F\left[U_{N}(T)\right] \geqslant F[U(T)]
$$

где $U_{N}(T) \rightarrow U(T)$ слабо в $Y^{\prime}$ по лемме 3.9 .

Соотношение (3.17) называется слабой полунепрерывностью снизу функционала $F$ и вьполняется для непрерывных локально ограниченных выпуклых функционалов [14; гл. III, $\S 8$, п. 8.4, теорема 8.10]. Непрерывность и локальная ограниченность рассматриваемого функционала $F$ уже отмечалась выше. Его выпуклость есть следствие выпуклости функции $\gamma(U)$, через которую он определяется $[15 ;$ гл. 9, 2 , п. 2.2, следствие 2.1]. Тем самым выполнены все условия для соотношения (3.17), которое соответствует п. (b) леммы, что завершает ее доказательстBO.

\section{§4. Предельный переход}

В предыдущем параграфе созданы предпосылки для доказательства следующего утверждения.

ТЕОРемА 4.1. Пусть выполнены условия теоремы 1.1, а также дополнительное условие $4 B$, имеющее вид: $B^{\prime}(u) \geqslant c_{0}>0$. Тогда верно заключение теоремы 1.1 о существовании решения задачи (1.1).

ДокАЗАТЕЛЬСТво. При дополнительном условии $4 B$ в предыдущем параграфе построена последовательность функций $u_{N}$ и связанная с ней последовательность $U_{N}=B u_{N}$. Для них установлены следующие факты:

$$
u_{N} \rightarrow u \text { слабо в } \mathscr{Z}, \quad A u_{N} \rightarrow W \text { слабо в } \mathscr{Z}^{\prime} .
$$

Кроме того, определена функция $U(t)=B u(t)$, которая является непрерывной и принимает значения в пространстве $Y^{\prime}$, наделенном слабой топологией, причем $B u(0)=B u_{0}$. Это доказывает пп. (a) и (d) теоремы 1.1. Далее, по лемме 3.8 для функции $U(t)$ определена производная $U^{\prime}(t)=\frac{\partial}{\partial t} B u$, которая удовлетворяет тождеству

$$
W+\frac{\partial}{\partial t} B u=f
$$

как равенству в пространстве $\mathscr{Z}^{\prime}$. Остается убедиться, что $W=A u$. В качестве вспомогательного шага вычислим произведение $\left\langle A u_{N}, u_{N}\right\rangle$. Используя тождество (3.8) для $e=u_{N}(t)$, после интегрирования по отрезку $[0, T]$ получаем

$$
\left\langle A u_{N}, u_{N}\right\rangle=\left\langle f, u_{N}\right\rangle-\left\langle U_{N}^{\prime}, u_{N}\right\rangle \text {. }
$$


Для произведения $\left\langle U_{N}^{\prime}, u_{N}\right\rangle$ применим лемму 3.3, что дает

$$
\left\langle A u_{N}, u_{N}\right\rangle=\left\langle f, u_{N}\right\rangle-\int_{\Omega} \Gamma\left[u_{N}(T, x)\right] d x+\int_{\Omega} \Gamma\left[u_{N}(0, x)\right] d x .
$$

Используя соотношения леммы 3.11 , получаем

$$
\begin{aligned}
\varlimsup_{N \rightarrow \infty}\left\langle A u_{N}, u_{N}\right\rangle & \leqslant\langle f, u\rangle-\int_{\Omega} \Gamma[u(T, x)] d x+\int_{\Omega} \Gamma[u(0, x)] d x \\
& =\langle f, u\rangle-\left\langle U^{\prime}, u\right\rangle=\langle W, u\rangle .
\end{aligned}
$$

Итак,

$$
\varlimsup_{N \rightarrow \infty}\left\langle A u_{N}, u_{N}\right\rangle \leqslant\langle W, u\rangle .
$$

Напомним одно свойство монотонных операторов [5; гл. $3, \S 1$, лемма 1.3]. Если выполнены соотношения (4.1) и (4.3) и при этом оператор $A$ является непрерывным и монотонным, то справедливо равенство $W=A u$. Подставляя его в (4.2), получаем тождество $A u+\frac{\partial}{\partial t} B u=f$, которое доказывает пп. (b) и (c) теоремы 1.1, что завершает доказательство теоремы 4.1.

Остается избавиться от дополнительного условия $4 B$ этой теоремы.

ДоКАЗАТЕЛЬСТВО ТЕОРЕМЫ 1.1. Итак, пусть выполнены только условия теоремы 1.1. В частности, функция $B(u)$ считается монотонно возрастающей. Отсюда следует, что ее производная неотрицательна. Введем для каждого $\varepsilon>0$ новую функцию $B_{\varepsilon}(u)=B(u)+\varepsilon u$. Тогда очевидно, что $B_{\varepsilon}^{\prime}(u) \geqslant \varepsilon>0$, т.е. для функции $B_{\varepsilon}(u)$ выполнено дополнительное условие $4 B$ при сохранении остальных условий $1 B-3 B$, и потому задача

$$
A u_{\varepsilon}+\frac{\partial}{\partial t} B_{\varepsilon} u_{\varepsilon}=f, \quad B u_{\varepsilon}(0)=B u_{0}
$$

имеет решение $u_{\varepsilon}$ со свойствами, описанными в теореме 1.1. Устремим $\varepsilon$ к нулю и убедимся, что предельная функция определена и дает решение задачи (1.1). Для этого оценим функции $u_{\varepsilon}$ так же, как последовательность $u_{N}$. Стоит отметить, что дополнительное условие $B^{\prime}(u) \geqslant c_{0}>0$ было использовано только при доказательстве существования функций $u_{N}$ и не применялось для вывода оценок на эти функции.

Проверим основные этапы этого нового доказательства. Напомним, что тождество (4.4) рассматривается как равенство в пространстве $\mathscr{Z} \prime$. В частности, это тождество можно умножать на функцию $u_{\varepsilon} \in \mathscr{Z}$, что приводит к равенству

$$
\begin{aligned}
\left\langle A u_{\varepsilon}, u_{\varepsilon}\right\rangle+\int_{\Omega} \Gamma\left[u_{\varepsilon}(T, x)\right] d x- & \int_{\Omega} \Gamma\left[u_{\varepsilon}(0, x)\right] d x \\
& +\frac{\varepsilon}{2} \int_{\Omega} u_{\varepsilon}^{2}(T, x) d x-\frac{\varepsilon}{2} \int_{\Omega} u_{0}^{2}(x) d x=\left\langle f, u_{\varepsilon}\right\rangle .
\end{aligned}
$$

Отсюда по аналогии с леммами 3.2 и 3.3 получаем оценки

$$
\begin{gathered}
\sup _{t}\left\|u_{\varepsilon}\right\|_{Y} \leqslant C, \quad\left\|u_{\varepsilon}\right\|_{\mathscr{Z}} \leqslant C, \\
\left\|A u_{\varepsilon}\right\|_{\mathscr{Z}^{\prime}} \leqslant X, \quad \sup _{t}\left\|B u_{\varepsilon}\right\|_{Y^{\prime}} \leqslant C .
\end{gathered}
$$


Это обеспечивает соответствующие слабые сходимости, которые представим в виде

$$
\begin{gathered}
u_{\varepsilon} \rightarrow u \text { слабо в } \mathscr{Z}, \quad A u_{\varepsilon} \rightarrow W \text { слабо в } \mathscr{Z}^{\prime}, \\
U_{\varepsilon} \equiv B_{\varepsilon}\left(u_{\varepsilon}\right) \rightarrow W_{0} \text { *-слабо в } L^{\infty}\left(0, T ; Y^{\prime}\right) .
\end{gathered}
$$

Таких сходимостей недостаточно для предельного перехода в уравнениях (4.4), поэтому стремимся получить еще сходимость последовательности $u_{\varepsilon}$ почти всюду в $Q$. Для этого составим произведение

$$
\left(\Delta_{h} U_{\varepsilon}, \Delta_{h} u_{\varepsilon}\right)=\left(U_{\varepsilon}(t+h)-U_{\varepsilon}(t), u_{\varepsilon}(t+h)-u_{\varepsilon}(t)\right)
$$

Отсюда по аналогии с леммой 3.5 получаем оценку снизу

$$
\left(\Delta_{h} U_{\varepsilon}, \Delta_{h} u_{\varepsilon}\right) \geqslant c_{2}\left\|u_{\varepsilon}(t+h)-u_{\varepsilon}(t)\right\|_{Y}^{r} .
$$

Одновременно рассматриваемое произведение можно оценить сверху, представив его в виде

$$
\left(\Delta_{h} U_{\varepsilon}, \Delta_{h} u_{\varepsilon}\right)=\int_{t}^{t+h}\left(U_{\varepsilon}^{\prime}(\tau), \Delta_{h} u_{\varepsilon}\right) d \tau .
$$

Поступая далее по аналогии с леммами $3.4,3.5$ и учитывая равномерные оценки (4.5), приходим к неравенству

$$
\left\|u_{\varepsilon}(t+h)-u_{\varepsilon}(t)\right\|_{\mathscr{Y}} \leqslant C h^{\beta}, \quad \beta>0 .
$$

Это дает возможность применить теорему 2.1 о компактности, из которой вытекает, что

$$
\begin{gathered}
u_{\varepsilon} \rightarrow u \text { п.в. в } Q, \quad u \in \mathscr{Z} \cap L^{\infty}(0, T ; Y), \\
U_{\varepsilon} \rightarrow U=B u \text { п.в. в } Q .
\end{gathered}
$$

По аналогии с леммой 3.8 убеждаемся, что определена производная

$$
U^{\prime}(t)=\frac{\partial}{\partial t} B u(t)
$$

как элемент пространства $\mathscr{D}^{\prime}\left(0, T ; Y^{\prime}\right)$ и при этом

$$
W+U^{\prime}=f
$$

Отсюда, в частности, следует, что $U^{\prime} \in \mathscr{Z}^{\prime}$. Кроме того,

$$
\begin{gathered}
U_{\varepsilon}(0) \rightarrow U(0)=B u_{0} \text { в } Y^{\prime}, \\
U_{\varepsilon}(T) \rightarrow U(T) \text { слабо в } Y^{\prime} .
\end{gathered}
$$

Это дает возможность по аналогии с доказательством теоремы 4.1 получить соотношение

$$
\varlimsup_{\varepsilon \rightarrow 0}\left\langle A u_{\varepsilon}, u_{\varepsilon}\right\rangle \leqslant\langle W, u\rangle .
$$

Здесь элементы $W, u$ взяты из (4.6). Так как $A$ - непрерывный монотонньй оператор, то из (4.8) вытекает, что $W=A u$. Подставляя это равенство в (4.7), получаем тождество $A u+\frac{\partial}{\partial t} B u=f$, которое показывает, что построенная функция $u(t, x)$ дает решение уравнения (1.1). В ходе доказательства были установлены остальные свойства этой функции, сформулированные в теореме 1.1, что завершает доказательство теоремы. 


\section{§5. Приложения к параболическим уравнениям высокого порядка}

Сформулированная в $\S 1$ теорема 1.1 достаточно легко применяется для доказательства разрешимости нелинейных параболических уравнений вида

$$
\begin{gathered}
\sum_{|\alpha| \leqslant m} D^{\alpha} A_{\alpha}\left(t, x, u, \ldots, D^{m} u\right)+\beta(u) \frac{\partial u}{\partial t}=f \\
u(0, x)=u_{0}(x), \quad m \geqslant 1 .
\end{gathered}
$$

Здесь $\alpha=\left(\alpha_{1}, \ldots, \alpha_{n}\right)$ - мультииндекс из целых неотрицательных чисел; $|\alpha|=$ $\alpha_{1}+\cdots+\alpha_{n} ; D^{\alpha}=D_{1}^{\alpha_{1}} \cdots D_{n}^{\alpha_{n}} ; D_{i}=\partial / \partial x_{i}(i=1, \ldots, n)$. Через $D^{k} u$ обозначается вектор из частных производных $D^{\alpha} u$ с условием $|\alpha|=k$. Уравнение рассматривается в ограниченной области $\Omega \in \mathbb{R}^{n}, n \geqslant 1$, с достаточно гладкой границей $\partial \Omega$, обеспечивающей применимость теорем вложения Соболева. Дифференциальное уравнение (5.1) дополняется граничными условиями, которые выделяют в пространстве $W_{p}^{m}(\Omega)$ с некоторым $p>1$ замкнутое подпространство $X$. Предполагается, что

$$
\stackrel{\circ}{W}_{p}^{m}(\Omega) \subset X \subset W_{p}^{m}(\Omega)
$$

Напомним некоторые возможности. Случай $X=\stackrel{\circ}{W_{p}^{m}}(\Omega)$ соответствует однородньпм условиям Дирихле

$$
\left.D^{\gamma} u\right|_{\partial \Omega}=0 \quad(|\gamma| \leqslant m-1)
$$

В качестве плотного множества гладких функций можно выбрать $X_{0}=C_{0}^{m}(\Omega)$.

Если же считать $X=W_{p}^{m}(\Omega)$, то в качестве дополнительных условий получаются так назьваемые естественные граничные условия, выписывать которые довольно затруднительно. В случае $m=1$ они приводят к нелинейному аналогу граничных условий Неймана. В качестве плотного в $X$ множества гладких функций можно выбрать $X_{0}=C^{m}(\bar{\Omega})$. В целом следует признать, что детальное описание допустимых граничных условий еще не разработано.

Итак, считаем, что граничные условия порождают банахово пространство $X$, удовлетворяюшее (5.2). По нему строим пространство $\mathscr{X}=L^{p}(0, T ; X)$ абстрактных функций, определенных на отрезке $[0, T]$ и принимающих значения в $X$. Предполагается, что операторы суперпозиции

$$
A_{\alpha} u \equiv A_{\alpha}\left(t, x, u(t, x), \ldots, D^{m} u(t, x)\right)
$$

переводят каждую функцию $u \in \mathscr{X}$ в элемент из $L^{p^{\prime}}(Q)=L^{p^{\prime}}\left(0, T ; L^{p^{\prime}}(\Omega)\right)$. По известной теореме об операторе суперпозиции [2; гл. I, $\S 2$, теорема 2.1] это обеспечивает непрерывность и ограниченность операторов $A_{\alpha}: \mathscr{X} \rightarrow L^{p^{\prime}}(Q)$, что достаточно для непрерьвности и ограниченности оператора

$$
A u=\sum_{|\alpha| \leqslant m}(-1)^{|\alpha|} D^{\alpha} A_{\alpha}\left(t, x, u, \ldots, D^{m} u\right)
$$

рассматриваемого как оператор из $\mathscr{X}$ в $\mathscr{X}^{\prime}$. Именно этот оператор предполагается монотонньм и коэрцитивньм в смысле условий $1 A-2 A \S 1$. Напомним наиболее 
известные примеры дифференциальных выражений, порождающих такие операторы:

$$
\begin{aligned}
& A u=\sum_{|\alpha|=m}(-1)^{m} D^{\alpha}\left[a_{\alpha}(t, x)\left|D^{\alpha} u\right|^{p-2} D^{\alpha} u\right] \\
& A u=\sum_{|\alpha|=m}(-1)^{m} D^{\alpha}\left[a_{\alpha}(t, x)\left|D^{m} u\right|^{p-2} D^{\alpha} u\right] .
\end{aligned}
$$

Здесь функции $a_{\alpha}(t, x) \in L^{\infty}(Q)$ и удовлетворяют условию $a_{\alpha}(t, x) \geqslant c_{0}>0$. Если указанные дифференциальные выражения дополнить одним из описанных ранее граничных условий, то получится монотонный коэрцитивный оператор, действующий из пространства $\mathscr{X}=L^{p}(0, T ; X)$ в $\mathscr{X}^{\prime}$.

Перейдем к описанию допустимых функций $\beta(u)$. Необходимые условия на них - это неотрицательность и степенной рост при $|u| \rightarrow \infty$. Наиболее простой функцией, подходящей под указанные условия, является $\beta(u)=|u|^{\nu}, \nu>0$. По $\beta(u)$ строим функции

$$
\begin{aligned}
& B(u)=\int_{0}^{u} \beta(s) d s=(\nu+1)^{-1}|u|^{\nu} u, \\
& \Gamma(u)=\int_{0}^{u} s \beta(s) d s=(\nu+2)^{-1}|u|^{\nu+2},
\end{aligned}
$$

для которых очевидно выполнение условий $1 B-3 B$ с показателем $r=\nu+2$. С указанньм $r$ вводим пространство $Y=L^{r}(\Omega)$, участвуюшее в формулировке теоремы 1.1.

В приведенном примере функция $\beta(u)$ имеет точно один нуль. Это совершенно не обязательно. Например, подходит функция $\beta(u)=|u|^{\nu}|u-1|^{\mu}$, где $\nu, \mu>0$. Непосредственным обобщением последнего примера является функция

$$
\beta(u)=\left|u-b_{1}\right|^{\nu_{1}}\left|u-b_{2}\right|^{\nu_{2}} \cdots\left|u-b_{l}\right|^{\nu_{l}},
$$

где числа $b_{i}(i=1, \ldots, l)$ произвольны, а степени $\nu_{i}$ положительны. Тогда число $r=2+\sum_{i=1}^{l} \nu_{i}$. Очевидно, что подходят также линейные комбинации функций указанной структуры с положительньми коэффициентами.

До сих пор не использовалась возможность сузить область определения оператора $A$ на пространство $\mathscr{X} \cap \mathscr{Y}$. Это имеет смысл при малых степенях $p$ и больших $r$, когда пространство $X=W_{p}^{m}(\Omega)$ не вкладывается в $Y=L^{r}(\Omega)$. Тогда есть возможность увеличить степени младших составляющих оператора $A$. Например, дифференциальное выражение

$$
A u=\sum_{|\alpha|=m}(-1)^{m} D^{\alpha}\left(\left|D^{\alpha} u\right|^{p-2} D^{\alpha} u\right)+|u|^{s-2} u
$$

порождает непрерывный монотонный оператор из $\mathscr{X}=L^{p}\left(0, T ; \stackrel{\circ}{W_{p}^{m}}(\Omega)\right)$ в $\mathscr{X}^{\prime}$ при условии, что $s \leqslant p$. Однако этот оператор остается непрерьвньп при $s>p$ на множестве $\mathscr{X} \cap \mathscr{Y}$, если $s \leqslant r$. 
В заключение приведем пример задачи, для которой выполняются все условия теоремы существования решения:

$$
\begin{gathered}
\sum_{|\alpha|=m}(-1)^{m} D^{\alpha}\left(\left|D^{\alpha} u\right|^{p-2} D^{\alpha} u\right)+|u|^{r-2} \frac{\partial u}{\partial t}=f(t, x), \\
u(0, x)=u_{0}(x),\left.\quad D^{\gamma} u\right|_{\partial \Omega}=0 \quad(|\gamma| \leqslant m-1) .
\end{gathered}
$$

Здесь степени $p>1$ и $r \geqslant 2$ произвольны. Если $u_{0} \in L^{r}(\Omega), f \in L^{p^{\prime}}\left(0, T ; W_{p^{\prime}}^{-m}(\Omega)\right)$ $+L^{r^{\prime}}(Q)$, то по теореме 1.1 эта задача имеет решение $u(t, x)$ с указанными в теореме свойствами. Напомним, что для уравнений второго порядка, т.е. для случая $m=1$, теория разрешимости указанной задачи была разработана Равьяром и детально изложена в монографиии Лионса [1; гл. $4, \S 1]$.

\section{Список литературы}

1. Лионс Ж.-Л. Некоторые методы решения нелинейных краевых задач. М.: Мир, 1972.

2. Скрыпник И. В. Методы исследования нелинейных эллиптических граничных задач. М.: Наука, 1990.

3. Дубинский Ю. А. Нелинейные эллиптические и параболические уравнения // Совр. пробл. матем. Т. 9. М.: ВИНИТИ, 1976. С. 5-130.

4. Дубинский Ю. А. Нелинейные эллиптические и параболические уравнения // Совр. пробл. матем. Т. 37. М.: ВИНИТИ, 1990. С. 89-166.

5. Гаевский X., Грегер K., Захариас $K$. Нелинейные операторные уравнения и операторные дифференциальные уравнения. М.: Наука, 1978.

6. Иванов А. В. Квазилинейные параболические уравнения, допускающие двойное вырождение // Алгебра и анализ. 1992. Т. 4. №6. С. 114-130.

7. Иванов A. В. Гёльдеровские оценки для уравнений типа быстрой диффузии // Алгебра и анализ. 1994. Т. 6. № 4. С. 101-142.

8. Иванов А. В., Мкртычян П. З., Яегер В. Сушествование и единственность регулярного решения первой начально-краевой задачи для некоторого класса дважды нелинейных параболических уравнений // Записки научн. сем. ПОМИ. 1994. Т. 213. С. 48-65.

9. Лаптев Г.И. Слабые решения квазилинейных параболических уравнений второго порядка с двойной нелинейностью // Матем. сб. 1997. Т. 188. № 9. С. 83-112.

10. Bernis $F$. Existence results for doubly nonlinear higher order parabolic equations on unbounded domains // Math. Ann. 1988. V. 279. P. 373-394.

11. Bernis F. Finite speed of propagation and asymptotic rates for some nonlinear higher order parabolic equations with absorption // Proc. Roy. Soc. Edinburgh. Sect. A. 1986. V. 104. № 1-2. P. 1-19.

12. Grange $O$. ., Mignot $F$. Sur la résolution d'une équation et d'une inéquation paraboliques non lineaires // J. Funct. Anal. 1972. V. 11. P. 77-92.

13. Беккенбах Э., Беллман Р. Неравенства. М.: Мир, 1965.

14. Вайнберг М. М. Вариационный метод и методмонотонных операторов. М.: Наука, 1972.

15. Экланд И., Темам Р. Выпуклый анализ и вариационные проблемы. М.: Мир, 1979.

Тульский государственньй университет

E-mail : laptev@home.tula.net

Поступила в редакцию

13.03.1999 